\title{
Resistência do couro de tilápia do Nilo submetido a diferentes processos de curtimento
}

\author{
Neu, D.H. ${ }^{1}$; Dallagnol, J.M. ${ }^{3}$; Klein, S. ${ }^{2}$; Maluf, M.L.F. ${ }^{4}$; Franco, M.L.S. ${ }^{3}$ e Boscolo, W.R. ${ }^{4}$
}

Universidade Federal da Grande Dourados-UFGD. Brasil.

2Instituto Federal do Mato Grosso do Sul-IFMS. Brasil.

${ }^{3}$ Universidade Estadual de Maringá-UEM. Brasil.

${ }^{4}$ Universidade Estadual do Oeste do Paraná-UNIOESTE. Brasil.

\section{PALAVRAS CHAVE ADICIONAIS}

Curtimento de peles.

Método estático.

Oreochromis niloticus.

Região corporal.

Taninos.

\section{RESUMO}

O objetivo foi determinar a resistência de couros de tilápia do Nilo que foram submetidas a dois tipos de curtimento, estático e em movimento, com e sem a extração da hipoderme. As unidades empregadas na experiência foram dois baldes (estático) e um fulão (movimento). Foram avaliados os parâmetros de resistência ao rasgamento progressivo, tração e alongamento, para os tratamentos: Balde com carne região dorsal, Balde com carne região ventral, Balde sem carne região dorsal, Balde sem carne região ventral, Fulão com carne região dorsal, Fulão com carne região ventral, Fulão sem carne região dorsal e Fulão sem carne região ventral. $O$ curtimento em fulão diferiu $(p<0,05)$ para rasgamento progressivo, tração e alongamento no corpo de prova maior, enquanto que o corpo de prova menor diferiu apenas para tração. No método (com e sem extração da hipoderme), houve diferença apenas no corpo de prova menor para tração. A região corporal (dorsal e ventral) diferiu $(p<0,05)$ para tração no corpo de prova maior e alongamento no corpo de prova menor. O melhor resultado foi apresentado pelo Fulão sem carne região dorsal $(102,21$ $\mathrm{N} . \mathrm{mm}^{-1} ; 16,00 \mathrm{~N} \cdot \mathrm{mm}^{-2}$ e $85,50 \%$ para rasgamento, tração e alongamento, respectivamente, para o corpo de prova maior). O curtimento no fulão proporciona superior qualidade ao couro, e o método não influenciou nas avaliações com exceção do corpo de prova menor.

\section{Nile Tilapia leather resistance submitted to different tanning processes}

\section{SUMMARY}

The aim was to determine the resistance of Nile tilapia leather which underwent two types of tanning, static and moving, with and without hypodermis extraction. Two buckets (static) and a drum (moving) were the units used in the experiment. We evaluated resistance to gradual tearing, traction, stretching parameters for the following treatments: Bucket with dorsal meat, Bucket with ventral region meat, Bucket without dorsal meat, Bucket without ventral meat, Drum with dorsal meat, Drum with ventral region meat, Drum without dorsal meat and Drum without ventral region meat. Tanning in drum differed $(p<0.05)$ for gradual tearing, traction and stretching in the larger specimen, while the smaller specimen differed only for traction. In the method (with and without hypodermis extraction), the only difference was in the smaller specimen for traction. The body region (dorsal and ventral) differed $(p<0.05)$ for traction in the larger specimen, and for stretching in the smaller specimen. The best result was presented by the Drum without dorsal meat $\left(102.21 \mathrm{~N} . \mathrm{mm}^{-1} ; 16.00 \mathrm{~N} . \mathrm{mm}^{-2}\right.$ and $85.50 \%$ for tearing, traction and stretching, respectively, in the larger specimen). Tanning in Drum provides superior quality to leather, and the method did not influence the assessments excepted the smaller specimen.

\section{INFORMACIÓN}

Cronología del artículo.

Recibido/Received: 28.5.2015

Aceptado/Accepted: 14.8.2015

On-line: 16.9.2015

Correspondencia a los autores/Contact e-mail:

jack.e.md@hotmail.com

\section{INTRODUÇÃO}

A aquicultura brasileira vem em crescimento constante, sendo a atividade agropecuária com expressivo incremento de produção, acima de $40 \%$ (MPA, 2010). Porém, a maior parte dessa produção está alavancada em principalmente uma espécie, a tilápia do Nilo. As tilápias foram introduzidas no Brasil a partir da década de 70 através do Departamento Nacional de Obras Contra as Secas (DNOCS), e por encontrar clima propício, além de nos últimos anos, surgirem tecnologias 
para o manejo e a nutrição, a mesma se adaptou com extrema facilidade.

Um entrave na utilização dessa espécie é a sua subutilização. Geralmente, seu produto final (filé) tem um rendimento médio de 30 a $40 \%$, e quando não possui qualquer tipo de processamento secundário, 70 a $60 \%$ podem acabar se transformando em resíduos (Maluf e Hilbig, 2010). Geralmente, as peles tem um rendimento entre 4,5 e 10\% (Souza, 2007), com média de 7,5\% (Contreras-Guzman, 1994). Fazendo uma estimação dos resíduos da pele apenas de tilápias geradas durante o ano de 2009 no Brasil, com rendimento médio de 7,5\%, das 132.957 toneladas produzidas (MPA, 2010), 9.971 são de peles. De acordo com Souza et al. (2003a), essas peles, muitas vezes, são moídas junto com os demais resíduos (vísceras, cabeças, etc.) e fornecidas como fonte de alimento.

Contudo, a não utilização desses produtos é um desperdício, pois sua aplicação em indústrias de vestuários ou artefatos em geral pode proporcionar uma importante fonte de rendimento, devido a sua transformação e agregação de valor (Souza et al., 2006). A pele é um produto nobre que tem como característica peculiar a resistência (Souza et al., 2003a).

Diversos são os fatores que podem afetar a resistência do couro de peixes, dentre eles, técnicas aplicadas no curtimento e engraxe, (Godoy et al., 2010). Durante as etapas da transformação da pele em couro, a primeira delas é a eliminação dos restos de carne que ficam aderentes sob a pele. Esse descarne necessita de tempo e pode resultar no aumento da mão de obra.

Nesse sentido, o objetivo com este trabalho foi verificar a resistência e a composição química do couro de tilápias do Nilo, submetidos ao curtimento em duas estruturas (estática e em movimento), dois processos (com carne e descarnadas) e em duas regiões corporais (dorsal e ventral).

\section{MATERIAL E MÉTODOS}

A experiência foi realizada no Laboratório de Processamento de Peles da Universidade Estadual do Oeste do Paraná - Unioeste, campus Toledo. As peles de tilápia foram adquiridas num frigorífico do mesmo município. Os peixes foram abatidos e filetados a partir de máquinas específicas.

Os tratamentos experimentais consistiram no curtimento das peles de tilápia do Nilo (Oreochromis niloticus) em equipamentos em movimento (fulão) e estático (baldes), essas peles anteriormente foram submetidas ao processo de extração da carne aderente ao couro (com carne) e sem o procedimento de extração da carne (com carne). Para tanto as mesmas foram armazenadas numa solução saturada de salmoura (7 Baumé).

O trabalho de descarne procedeu-se em mesa plana com o auxílio de colheres de metal, empregando se o movimento contínuo da parte anterior para a posterior das peles. Foi utilizado um fulão com capacidade de 60 quilos, em que as peles com carne e sem carne foram curtidas. Para a diferenciação, as peles descarnadas foram identificadas com pequenos cortes na região posterior, local escolhido pela fácil visualização e pela não interferência dos cortes de prova. Também foram utilizados dois baldes com capacidade de 30 quilos. Em cada balde havia um diferente tratamento, com carne e sem carne. A cada etapa do processo de curtimento as peles dos baldes foram retiradas para verificar a variação do seu peso e elaboração de um perfil, também foram retiradas 200 gramas de cada amostra, identificadas e congeladas para posterior análises de composição físico-química.

As análises fisico-químicas analisadas foram: determinação de óxido de cromo (ABNT - NBR 11054, 1999a), determinação do $\mathrm{pH}$ e da cifra diferencial em extrato aquoso (ABNT - NBR 11030, 1997a) e determinação de substâncias extratíveis em diclorometano (ABNT - NBR 11054, 1999b). Essas análises foram realizadas pela BASF.

Para o curtimento, as peles passaram por diversos processos adaptados da metodologia proposta por Souza (2004): remolho, caleiro, desencalagem, purga, desengraxe, píquel, curtimento (10\% tanino vegetal), neutralização, recurtimento (1\% tanino sintético e $3 \%$ tanino vegetal), tingimento, engraxe e fixação, secagem e amaciamento.

Após a secagem, as peles foram levadas para o Laboratório de processamento de peles de peixes e de demais espécies de pequeno e médio porte do Departamento de Zootecnia da Universidade Estadual de Maringá - UEM, Maringá, para a realização dos testes físico mecânicos.

\section{ANÁLISE FÍSICO-MECÂNICA}

Foram utilizados 20 couros obtidos a partir das peles curtidas de cada um dos tratamentos: fulão e balde, com carne e descarnadas. 10 couros de cada tratamento foram analisados de cada uma das regiões corporais, dorsal e ventral. Os corpos de prova retirados com auxílio de um balancim (ABNT - NBR 11035, 1990) consistiam de uma amostra para rasgamento progressivo (ABNT - NBR 11055, 1997b) e duas amostras para resistência à tração e alongamento (ABNT - NBR 11041, 1997c) de diferentes tamanhos. As amostras analisadas foram retiradas no sentido longitudinal ao comprimento do peixe e identificadas.

A seguir, as amostras foram levadas ao laboratório, em ambiente climatizado a $23 \pm 2^{\circ} \mathrm{C}$ e umidade relativa do ar de $50 \pm 2 \%$, por um período de 24 horas (NBR $10455,1988)$. Os testes foram realizados com dinamômetro EMIC, com velocidade de afastamento entre cargas de $100 \pm 20 \mathrm{~mm} / \mathrm{mm}$. Antes de realizar os testes físico-mecânicos, determinaram-se as medidas de espessura das amostras, de acordo com a Associação Brasileira de Normas Técnicas (NBR 11062, 1997d).

\section{Procedimento estatístico}

Os testes estatísticos foram desenvolvidos de maneira trifatorial, em que a estrutura, o método e a região corporal eram os parâmetros categóricos (Estrutura $\times$ Método $\times$ Região), e os demais parâmetros avaliados, os dependentes. Foi realizada uma ANOVA fatorial em $5 \%$ de probabilidade pelo teste Tukey, através do programa computacional Statistic 7.1. (STATSOFT, 2005). 
Tabela I. Média dos resultados dos testes de resistência de rasgamento progressivo das peles de tilápia do Nilo submetidas ao curtimento com diferentes estruturas e métodos (Average results from gradual tearing resistance tests of Nile tilapia leather submitted to tanning with different structures and methods).

\begin{tabular}{|c|c|c|c|c|c|}
\hline Região corporal & Estrutura & Método & Espessura (mm) & Rasgo (N.mm $\left.{ }^{1}\right)$ & Força máxima $(\mathrm{N})$ \\
\hline Dorsal & Balde & Carne & $0,67^{b}$ & $62,20^{\mathrm{bc}}$ & $41,20^{b}$ \\
\hline Ventral & Balde & Carne & $0,77^{\mathrm{a}}$ & $58,15^{c}$ & $45,40^{b}$ \\
\hline Dorsal & Balde & Sem carne & $0,67^{b}$ & $66,95^{\mathrm{bc}}$ & $44,50^{b}$ \\
\hline Ventral & Balde & Sem carne & $0,70^{\mathrm{ab}}$ & $79,18^{a b c}$ & $53,80^{\mathrm{ab}}$ \\
\hline Dorsal & Fulão & Carne & $0,67^{b}$ & $80,82^{\mathrm{abc}}$ & $54,50^{\mathrm{ab}}$ \\
\hline Ventral & Fulão & Carne & $0,67^{\mathrm{b}}$ & $85,67^{\mathrm{ab}}$ & $57,22^{\mathrm{ab}}$ \\
\hline Dorsal & Fulão & Sem carne & $0,68^{a b}$ & $102,21^{\mathrm{a}}$ & $67,55^{a}$ \\
\hline Ventral & Fulão & Sem carne & $0,77^{a}$ & $64,55^{\mathrm{bc}}$ & $49,70^{b}$ \\
\hline \multicolumn{6}{|l|}{$p$} \\
\hline Estrutura & & & 0,82 & $<0,01^{*}$ & $<0,01^{*}$ \\
\hline Método & & & 0,68 & 0,13 & 0,12 \\
\hline Região corporal & & & $0,01^{*}$ & 0,15 & 0,88 \\
\hline Estrutura*Método & & & $0,02^{*}$ & 0,14 & 0,58 \\
\hline Estrutura*Região & & & 0,69 & $0,02^{*}$ & $0,01^{*}$ \\
\hline Método*Região & & & 0,84 & 0,13 & 0,17 \\
\hline Estrutura*Método*Região & & & 0,06 & $<0,01^{*}$ & $0,02^{*}$ \\
\hline C.V. $(\%)$ & & & 13,65 & 30,49 & 27,47 \\
\hline
\end{tabular}

Medias com letras distintas na mesma coluna diferem através do teste Tukey em $5 \%$ de probabilidade.

\section{RESULTADOS E DISCUSSÃO}

Para a avaliação do rasgamento progressivo dos corpos de prova ocorreram diferenças significativas entre os parâmetros de espessura $(\mathrm{mm})$ das peles para a região corporal, sendo a parte ventral mais espessa. $\mathrm{Na}$ resistência ao rasgo $\left(\mathrm{N} . \mathrm{mm}^{-1}\right)$ e força máxima $(\mathrm{N})$ aplicada aos couros, ocorreram diferenças significativas em relação à estrutura $(\mathrm{p}<0,05)$, interação da estrutura com a região corporal e interação entre todos os parâmetros avaliados, como pode ser observado na tabela I.

Com relação à espessura das peles nos corpos de prova submetidos ao rasgamento progressivo, os mesmos tiveram diferenças observadas para cada região corporal, dorsal e ventral. A espessura observada na presente experiência, é semelhante à espessura verificada por Godoy et al. (2010) para o couro de tilápias vermelhas, quando retirados os corpos de prova nos sentidos longitudinal e transversal, porém os autores não verificaram diferenças significativas. Franco et al. (2013) verificaram que a espessura do couro da tilápia é de $0,65 \mathrm{~mm}$, próximo ao descrito na presente pesquisa. Os autores destacam ainda que embora o processo de transformação de pele em couro seja complexo, não há valores descritos como padrão para o curtimento de pele de peixes.

Essa diferença em termos de espessura na região ventral também foi observada por Souza et al. (2003b) para o pacu (Piaractus mesopotamicus), em que, na mesma região a camada mais externa da derme apresenta fibras colágenas transversais à superfície da pele, tornando-a mais espessa pois as fibras estão próximas umas das outras. Contudo, na tilápia, a camada mais profunda da derme apresenta feixes de fibras colágenas finos, sobrepostas e cruzadas no sentido transver- sal à superfície da pele (Franco et al., 2013), por isso a espessura é inferior entre as espécies.

O maior valor de rasgamento progressivo encontrado foi de 102,21 N.mm ${ }^{-1}$, no grupo de couros que foram curtidos em fulão com a retirada do excesso de carne e na região dorsal. E o menor valor observado foi para o grupo de couros curtidos em balde sem a eliminação da carne e na região ventral da pele do peixe. De acordo com Souza et al. (2006) se houver maior quantidade de musculatura de filé aderida a pele, ocorre maior dificuldade no processo de curtimento. Possivelmente a estrutura onde foi realizado o curtimento contribuiu para que houvesse maior ação dos agentes curtentes e elevassem valores no rasgamento progressivo, contudo, vale ressaltar que a região corporal analisada também apresenta efeito.

Na presente experiência, foram utilizados taninos de origem vegetal e sintético como agente curtente, e o valor encontrado para o rasgo, no teste de rasgamento progressivo foi inferior ao relatado por Hilbig et al. (2013), isso pode ser explicado pelo fato das diferenças de tempo e concentração dos produtos no processo de curtimento, pois os agentes curtentes (taninos) utilizados em ambos os trabalhos eram os mesmos. Prado (2011) relata valores de rasgamento progressivo para o pacu acima de $63 \mathrm{~N} . \mathrm{mm}^{-1}$, enquanto o de tilápia acima de $40 \mathrm{~N} . \mathrm{mm}^{-1}$, assim como o observado no presente estudo. Essa diferença pode ser atribuída ao teor de hidroxiprolina no colágeno das diferentes espécies de peixes, interferindo na temperatura de retração ou encolhimento da pele, onde ocorre a ruptura das ligações de hidrogênio, e nos peixes de água quente, como o caso da tilápia, a temperatura de retração da pele é maior quando comparado a peixes de águas frias (Sanches e Araya, 1990 apud Souza, 2003c). Todavia, os resultados do presente estudo estão acima 
Tabela II. Média dos resultados dos testes de resistência de tração e alongamento das peles de tilápia do Nilo submetidas ao curtimento com diferentes estruturas e métodos no corpo de prova maior (Average results from traction and stretching resistance tests of Nile tilapia leather submitted to tanning with different structures and methods in the larger specimen).

\begin{tabular}{|c|c|c|c|c|c|c|}
\hline Região corporal & Estrutura & Método & Espessura $(\mathrm{mm})$ & Tração (N.mm²) & Força máxima (N) & Alongamento (\%) \\
\hline Dorsal & Balde & Carne & 0,72 & $11,74^{\mathrm{bcd}}$ & $83,10^{\mathrm{ab}}$ & $62,70^{\mathrm{b}}$ \\
\hline Ventral & Balde & Carne & 0,73 & $8,07^{d}$ & $60,50^{\mathrm{b}}$ & $74,10^{\mathrm{ab}}$ \\
\hline Dorsal & Balde & Sem carne & 0,73 & $12,22^{\mathrm{abc}}$ & $86,50^{\mathrm{ab}}$ & $61,80^{b}$ \\
\hline Ventral & Balde & Sem carne & 0,66 & $10,21^{\mathrm{cd}}$ & $68,20^{\mathrm{b}}$ & $65,60^{b}$ \\
\hline Dorsal & Fulão & Carne & 0,74 & $13,83^{a b c}$ & $100,50^{a}$ & $66,50^{\mathrm{ab}}$ \\
\hline Ventral & Fulão & Carne & 0,68 & $14,89^{\mathrm{ab}}$ & $101,20^{a}$ & $85,30^{\mathrm{a}}$ \\
\hline Dorsal & Fulão & Sem carne & 0,69 & $16,00^{\mathrm{a}}$ & $109,10^{a}$ & $85,50^{a}$ \\
\hline Ventral & Fulão & Sem carne & 0,67 & $14,61^{\mathrm{ab}}$ & $97,78^{a}$ & $77,67^{a}$ \\
\hline \multicolumn{7}{|l|}{$\mathrm{p}$} \\
\hline Estrutura & & & 0,34 & $<0,01^{*}$ & $<0,01^{*}$ & $<0,01^{*}$ \\
\hline Método & & & 0,06 & 0,07 & 0,35 & 0,91 \\
\hline Região corporal & & & 0,05 & $0,01^{*}$ & $<0,01^{*}$ & 0,15 \\
\hline Estrutura*Método & & & 1,00 & 0,76 & 0,73 & 0,25 \\
\hline Estrutura*Região & & & 0,65 & $0,03^{*}$ & 0,08 & 0,81 \\
\hline Método*Região & & & 0,47 & 0,75 & 0,65 & 0,06 \\
\hline Estrutura*Método*Região & & & 0,10 & 0,10 & 0,35 & 0,29 \\
\hline C.V. $(\%)$ & & & 10,62 & 28,63 & 27,78 & 29,44 \\
\hline
\end{tabular}

Medias com letras distintas na mesma coluna diferem através do teste Tukey em $5 \%$ de probabilidade.

de 40 N.mm-1 e como não há um padrão estabelecido, acredita-se que dentro dos limites adequados para o couro dessa espécie.

O teste de rasgamento progressivo confirma que na região dorsal, os couros que passaram pelo processamento de eliminação da carne aderente, ficaram mais resistentes tanto para as peles que foram curtidas em balde, quanto para as peles curtidas em fulão. Já na região ventral, os couros curtidos no fulão, em movimento, tiveram menor resistência que os mesmos curtidos com a presença de carne, relação inversa ao observado para os couros da mesma região ventral, porém que foram curtidos em balde.

Com relação aos testes de resistência à tração e alongamento do corpo de prova maior (tabela II), é possível verificar que não houve diferenças significativas na espessura das peles, porém para a tração $\left(\mathrm{N} . \mathrm{mm}^{-2}\right)$ e força máxima $(\mathrm{N})$ houve interação entre a estrutura utilizada no curtimento e região do corpo, e para o alongamento $(\%)$, a região do corpo foi determinante na verificação das diferenças $(p<0,05)$.

A estrutura em que as peles são curtidas pode definir maior resistência ao couro. A maior resistência a tração foi verificada para o grupo de peles que foram submetidas à extração da carne, curtidas em fulão e na região dorsal, com valor de $16,00 \mathrm{~N} . \mathrm{mm}^{-2}$, resistência superior ao reportado por Souza et al. (2006), também para a tilápia do Nilo. Por outro lado, as amostras menos resistentes a tração correspondem ao grupo de couros submetidos ao curtimento em baldes, sem a extração da carne e na região ventral, que tiveram valores médios de 8,07 N.mm².

A estrutura utilizada no curtimento, fulão e balde, são responsáveis pela influência da tração, força máxima e alongamento das peles. As maiores trações são alcançadas quando o curtimento é realizado em fulão, assim como, quando a maior força é aplicada. Isso deve-se ao fato de que, em movimento, os couros tornam-se mais resistentes, quando comparado ao curtimento em maneira estática.

Outro fator que pode contribuir para o aumento da resistência, é que a ação mecânica de movimento, faz com que a penetração dos agentes curtentes seja mais eficaz, neste caso os taninos vegetal e sintético, ao couro, e, portanto, os mesmos tornam-se mais resistentes. Outra vantagem do curtimento com movimentação, é que os couros tornam-se mais elásticos, atingindo até $85,50 \%$ de elasticidade na região dorsal quando curtidos em fulão, e com a retirada da carne aderente.

Prado (2011) relata que teor de hidroxiprolina no colágeno é diferente para as distintas espécies de peixes, e pacu e tambaqui apresentam quantidades de hidroxiprolina acima de $3 \%$, o que resulta em uma maior capacidade de resistência à tração, acima de 18,0 e 29,0 N.mm ${ }^{-2}$, respectivamente, enquanto a tração da tilápia é cerca de 11,0 N.mm ${ }^{-2}$.

Vieira et al. (2008) realizaram o curtimento de peles de peixe com o uso de taninos sintéticos, vegetais e sua combinação e verificaram que embora ocorra diferenças significativas em relação à tração, a sua combinação proporciona resistência à tração de 11,88 N.mm ${ }^{-2}$ e alongamento entre 63 e $75 \%$, valores esses que são inferiores ao encontrado no atual estudo quando comparados à tração e alongamento dos couros curtidos em fulão, mas similar aos valores observados para o curtimento em baldes. Contudo, os maiores valores de resistência à tração foi observado por Souza e Silva (2005), com valores acima de 30 N.mm ${ }^{-2}$ quando o curtimento ocorreu através da utilização de sais de cromo, que são os agentes curtentes mais utilizados. De toda forma, a resistência à tração suportada pelos couros de 
Tabela III. Média dos resultados dos testes de resistência de tração e alongamento das peles de tilápia do Nilo submetidas ao curtimento com diferentes estruturas e métodos no corpo de prova menor (Average results from traction and stretching resistance tests of Nile tilapia leather submitted to tanning with different structures and methods in the smaller specimen).

\begin{tabular}{|c|c|c|c|c|c|c|}
\hline Região corporal & Estrutura & Método & Espessura (mm) & Tração (N.mm²) & Força máxima $(\mathrm{N})$ & Alongamento (\%) \\
\hline Dorsal & Balde & Carne & 0,75 & $6,85^{\mathrm{bc}}$ & $51,70^{\mathrm{bc}}$ & $39,80^{\mathrm{abc}}$ \\
\hline Ventral & Balde & Carne & 0,79 & $6,42^{\mathrm{c}}$ & $50,44^{\text {bc }}$ & $30,44^{c}$ \\
\hline Dorsal & Balde & Sem carne & 0,74 & $7,21^{\mathrm{bc}}$ & $51,90^{\mathrm{bc}}$ & $40,30^{\mathrm{ab}}$ \\
\hline Ventral & Balde & Sem carne & 0,73 & $6,57^{c}$ & $43,10^{c}$ & $34,00^{\mathrm{bc}}$ \\
\hline Dorsal & Fulão & Carne & 0,76 & $8,11^{\mathrm{bc}}$ & $61,80^{\mathrm{ab}}$ & $42,70^{\mathrm{ab}}$ \\
\hline Ventral & Fulão & Carne & 0,71 & $8,91^{\mathrm{abc}}$ & $62,56^{\mathrm{ab}}$ & $40,67^{\mathrm{ab}}$ \\
\hline Dorsal & Fulão & Sem carne & 0,69 & $9,31^{\mathrm{ab}}$ & $63,80^{\mathrm{ab}}$ & $39,80^{\mathrm{abc}}$ \\
\hline Ventral & Fulão & Sem carne & 0,74 & $11,00^{\mathrm{a}}$ & $80,00^{a}$ & $45,00^{\mathrm{a}}$ \\
\hline \multicolumn{7}{|l|}{$p$} \\
\hline Estrutura & & & 0,17 & $<0,01^{*}$ & $<0,01^{*}$ & $<0,01^{*}$ \\
\hline Método & & & 0,23 & $0,02^{*}$ & 0,31 & 0,35 \\
\hline Região corporal & & & 0,76 & 0,38 & 0,57 & $0,03^{*}$ \\
\hline Estrutura*Método & & & 0,80 & 0,09 & $0,03^{*}$ & 0,66 \\
\hline Estrutura*Região & & & 0,76 & $0,03^{*}$ & $0,02^{*}$ & $0,02^{*}$ \\
\hline Método*Região & & & 0,67 & 0,68 & 0,51 & 0,08 \\
\hline Estrutura*Método*Região & & & 0,12 & 0,50 & 0,06 & 0,48 \\
\hline C.V. $(\%)$ & & & 12,71 & 28,70 & 28,67 & 19,44 \\
\hline
\end{tabular}

Medias com letras distintas na mesma coluna diferem através do teste Tukey em $5 \%$ de probabilidade.

tilápia do atual estudo, são elevados, principalmente, quando utilizado o fulão como estrutura.

Outra vantagem do curtimento no sistema em movimento é uma maior eficácia no tingimento das peles, caracterizando coloração homogênea, em contrapartida, em estruturas estáticas, o tingimento ocorreu desuniforme na coloração dos couros, constatado visualmente, com algumas falhas ao longo das amostras.

Possivelmente, no curtimento empregando o sistema estático não houve o contato dos agentes curtentes de forma homogênea o que esclarece a diferença encontrada para os parâmetros de tração, força máxima e alongamento entre as duas estruturas utilizadas.

Com um corpo de prova menor, a tração sofre influencia tanto da estrutura quanto do método aplicado ao seu processamento $(\mathrm{p}<0,05)$, o alongamento difere significativamente com relação à estrutura e à região corporal (tabela III).

Em termos numéricos, a tração, a força máxima e o alongamento dos corpos de prova dos couros de tilápia com tamanho menor também são inferiores, quando comparado ao corpo de prova com tamanho superior.

Entretanto, embora os números nos indiquem que o tamanho é definitivo para uma maior tração a ser aplicada e no maior alongamento adquirido, o comportamento dos corpos de prova com tamanhos diferentes são os mesmos. A resistência à tração é mais elevada nas amostras curtidas em fulão, bem como essa estrutura de curtimento proporciona maior elasticidade. Portanto, o movimento exercido pelo equipamento promove um curtimento mais eficaz refletindo na qualidade do produto final.

Porém, no corpo de prova menor, o método de extração da carne teve os maiores valores de resistência $(\mathrm{p}<0,05)$, já nos demais corpos de prova, não houve essa diferença, o que pode contribuir para diminuição no custo com mão de obra para a extração da camada hipodérmica.

De acordo com Hoinacki (1989), segundo os Niveles de Calidad Aceptables en la Indústria del Cuero de Organización de las Naciones Unidas para El Desarro1lo Industrial (1976), o couro bovino curtido ao cromo deve apresentar uma resistência à tração de no mínimo 9,80 N.mm² ${ }^{-2}$ uma elongação até a ruptura de no mínimo $60 \%$ e o rasgamento progressivo de 14,70 N.mm ${ }^{-1}$ para a confecção de vestuário. Estes valores foram atingidos, com exceção do tratamento realizado em balde, sem a retirada da carne aderida ao epitélio e na região ventral, em que foi observada uma resistência a tração inferior a 9,0 N.mm .

Com relação aos teores de óxido de cromo, teor de graxa, pH e cifra diferencial do couro da tilápia, os mesmos podem ser verificados na tabela IV.

O teor de óxido de cromo não foi determinado em função de o curtimento ser realizado com taninos. Os valores de $\mathrm{pH}$ foram 4,43 e 4,30 para os tratamentos em movimento e 4,17 e 3,77 para os estáticos. Para cifra diferencial foi 0,75 , apenas para o tratamento balde sem carne. Pois a cifra diferencial somente age como um critério para a presença de ácidos fortes livres ou bases em extrato aquoso com valores de $\mathrm{pH}$ abaixo de 4,0 ou acima de 10 . Os valores para $\mathrm{pH}$ e cifra diferencial devem ser no mínimo 3,5 e máximo 0,7 , respectivamente (Hoinacki, 1989). Dentre os tratamentos avaliados somente a cifra diferencial para balde sem carne apresentou resultado superior ao recomendado.

As substâncias extraíveis em diclorometano foram $11 \%$ para os tratamentos com movimento e $5 \%$ para os tratamentos estáticos. De acordo com Vademécum 
Tabela IV. Médias dos valores de determinação do óxido de cromo, teor de graxa, pH e cifra diferencial do couro da tilápia Oreochromis niloticus (Average values for determination of chromium oxide, grease content, $\mathrm{pH}$ and differential cipher of leather from Oreochromis niloticus tilapia).

\begin{tabular}{|c|c|c|c|c|}
\hline \multirow{2}{*}{ Parâmetros } & \multicolumn{4}{|c|}{ Estrutura/Métodos } \\
\hline & 1 & 2 & 3 & 4 \\
\hline Determinação do óxido de cromo $\left(\mathrm{Cr}_{2} \mathrm{O}_{3}\right)(\%)$ & Ausente & Ausente & Ausente & Ausente \\
\hline Determinação pH em extrato aquoso & 4,17 & 3,77 & 4,43 & 4,30 \\
\hline Determinação cifra diferencial em extrato aquoso & Ausente & 0,75 & Ausente & Ausente \\
\hline Determinação de substâncias extraíveis em diclorometano (\%) & 5,8 & 5,3 & 11,7 & 11,8 \\
\hline
\end{tabular}

1= Balde com carne; 2= Balde sem carne; 3= Fulão com carne; 4= Fulão sem carne.

(1995), para o couro ser empregado na confecção de vestuários, as substâncias extraíveis em diclorometano não devem ultrapassar de 16 a 18\%. Souza et al. (2006), avaliando técnica de curtimento e do método utilizado para remoção da pele da tilápia do Nilo observaram que a quantidade de substâncias extraíveis em diclorometano foi de $19,42 \%$ pela técnica com sais de cromo e de $20,02 \%$ pela técnica sem sais de cromo (bioleather). Devido aos couros dos baldes permanecerem em menor movimento durante o processo de acabamento, o engraxe pode não ser bem realizado, sem a penetração total dos óleos (Prado, 2011) e por isso os valores observados nos baldes resultou em uma faixa ligeiramente abaixo do encontrado para o fulão, que está acima de $11 \%$.

No cenário apresentado neste trabalho, o curtimento das peles de tilápia do Nilo apresenta boa resistência ao rasgamento progressivo, bem como a tração imposta pelo dinamômetro e os couros demonstraram boa flexibilidade, com alta margem de alongamento. Os mesmos podem ser submetidos a processos de industrialização com garantia de qualidade ao produto final. Mesmo com algumas diferenças em relação às estruturas utilizadas, o procedimento estático mostrouse efetivo.

\section{CONCLUSÃO}

O curtimento no fulão proporciona superior qualidade ao couro, apresentando melhor resistência ao rasgo, tração e alongamento, além do pH e substâncias extraíveis em diclorometano. $\mathrm{O}$ método não influenciou nas avaliações com exceção do corpo de prova menor. Portanto, recomenda-se o emprego do fulão sem a remoção da hipoderme.

\section{AGRADECIMENTOS}

A empresa de produtos químicos BASF S.A. pelo inestimável apoio, e ao Grupo de Estudo de Manejo na Aquicultura - GEMAq, pela disponibilidade do Laboratório de Processamento de Peles.

\section{BIBLIOGRAFIA}

Associação Brasileira de Normas Técnicas - ABNT. 1988. NBR 10455: climatização de materiais usados na fabricação de calçados e correlatos. Rio de Janeiro. pp. 1-2.

Associação Brasileira de Normas Técnicas - ABNT. 1990. NBR 11035 : corte de corpos-de-prova em couro. Rio de Janeiro. p. 1 .
Associação Brasileira de Normas Técnicas - ABNT. 1997a. NBR 11030 : couro - determinação de substâncias extraíveis com diclorometano (CH2Cl2). Rio de Janeiro. pp. 1-3.

Associação Brasileira de Normas Técnicas - ABNT. 1997b. NBR 11055 : couro - determinação da força de rasgamento progressivo. Rio de Janeiro. pp. 1-4.

Associação Brasileira de Normas Técnicas - ABNT. 1997c. NBR 11041 : couros - determinação da resistência à tração e alongamento. Rio de Janeiro. pp. 1-5.

Associação Brasileira de Normas Técnicas - ABNT. 1997d. NBR 11062 : determinação da espessura. Rio de Janeiro. pp. 1.

Associação Brasileira de Normas Técnicas - ABNT. 1999a. NBR 11054 : couros - determinação de óxido crômico (Cr2O3). Rio de Janeiro. pp.1-5.

Associação Brasileira de Normas Técnicas - ABNT. 1999b. NBR 11057 : couro - determinação do $\mathrm{pH}$ e da cifra diferencial. Rio de Janeiro. pp.1-3.

Contreras-Guzmán, E.S. 1994. Bioquímica de pescados e derivados. FUNEP. Jaboticabal. 409 pp.

Franco, M.L.R.S.; Franco, N.P.; Gasparino, E.; Dorado, D.M.; Prado, M. e Vesco, A.P.D. 2013. Comparação das peles de tilápia do Nilo, pacu e tambaqui: Histologia, composição e resistência. Arch Zootec, 62: 21-32.

Godoy, L.C.; Gasparino, E.; Franco, M.L.R.S.; Franco, N.P. e Dourado, D.M. 2010. Testes físico-mecânicos e físico-químico do couro da tilápia vermelha. Arqu Bras Med Vet Zootecn, 62: 475-480.

Hilbig, C.C.; Fockink, D.H.; Maluf, M.L.F.; Boscolo, W.R. e Feiden, A. 2013. Resistência do couro de tilápia e composição centesimal da pele nas operações de ribeira e curtimento. Sci Agrária Paranaensis, 12: $258-266$

Hoinacki, E. 1989. Peles e couros: origens, defeitos e industrialização. 2. ed. rev. e ampl. Henrique d'Ávila Bertaso. Porto Alegre.

Maluf, M.L.F. 2010. Curtimento ecológico de peles de peixes. GFM. Toledo. $42 \mathrm{pp}$.

Maluf, M.L.F. e Hilbig, C.C. 2010. Curtimento ecológico de peles de animais para agregação de valor através da confecção de artesanato. Varia Sci, 9: 75-79.

Ministério da Pesca e Aquicultura - MPA. 2010. Produção pesqueira e aquícola: Estatística 2008 e 2009. Brasília. 30 pp.

Prado, M. 2011. Características da pele de Pseudoplatystoma sp. submetida ao processamento de curtimento: morfologia, resistência físico-mêcanica e química. Dissertação (Mestrado em Zootecnia). Universidade Estadual de Maringá. Maringá. PR. 74 pp.

Souza, M.L.R.; Dourado, D.M.; Machado, S.D.; Buccini, D.F.; Jardim, M.I.A.; Matias, R.; Correia, C. e Ferreira, I.C. 2003a. Análise da pele de três espécies de peixes: histologia, morfometria e testes de resistência. Rev Bras Zootecn, 32: 1551-1559.

Souza, M.L.R.; Ganeco, L.N.; Nakaghi, L.S.O.; Faria, R.H.S.; Wagner, P.M.; Povh, J.A. e Ferreira, I.C. 2003b. Histologia da pele do pacu (Piaractus mesopotamicus) e testes de resistência do couro. Acta Sci Anim Sci, 25: 37-44.

Souza, M.L.R. 2003c. Tecnologia para peles de peixes: processo de curtimento. In: Congreso Nacional de Investigaciones Acuícolas IV Muestra Comercial de Acuicultura. Bogotá. 
Souza, M.L.R. 2004. Tecnologia para processamento das peles de peixe. Eduem. Maringá. (Coleção Fundamentum, 11).

Souza, M.L.R. e Silva, L.O. 2005. Efeito de técnicas de curtimento sobre a resistência do couro da tilápia do Nilo (Oreochromis niloticus L.). Acta Sci Anim Sci, 27: 535-540.

Souza, M.L.R.; Casaca, J.M.; Nakaghi, L.S.O.; Franco, N.P.; Silva, L.O.; Dourado, D.M. e Viegas, E.M.M. 2006. Efeito da técnica de curtimento e do método utilizado para remoção da pele da tilápia do Nilo sobre as características de resistência do couro. Rev Bras Zootecn, 35: 1273-1280.
Souza, M.L.R. 2007. Curtimento de pele de tilápia. In: Boscolo, W.R.; Feiden, A. Industrialização de tilápias. GFM. Toledo. pp. 185-223.

Statsoft, Inc. 2005. Statistica (data analysis software system), version 7.1. www.statsoft.com.

Vademécum. 1995. Para el técnico em curtición. 3.ed.rev. Basf. Ludwigshanfen. $477 \mathrm{pp}$.

Vieira, A.M.; Kachba, Y.R.; Souza Franco, M.L.R.; Oliveira, K.F.; Godoy, L.C. e Gasparino, E. 2008. Curtimento de peles de peixe com taninos vegetal e sintético. Acta Sci Anim Sci, 42: 359-363. 
